\title{
Developmental stochasticity and variation in floral phyllotaxis
}

\author{
Miho Stephanie Kitazawa ${ }^{1}$
}

Received: 16 September 2020 / Accepted: 16 March 2021 / Published online: 5 April 2021

(c) The Author(s) 2021

\begin{abstract}
Floral phyllotaxis is a relatively robust phenotype; trimerous and pentamerous arrangements are widely observed in monocots and core eudicots. Conversely, it also shows variability in some angiosperm clades such as 'ANA' grade (Amborellales, Nymphaeales, and Austrobaileyales), magnoliids, and Ranunculales. Regardless of the phylogenetic relationship, however, phyllotactic pattern formation appears to be a common process. What are the causes of the variability in floral phyllotaxis and how has the variation of floral phyllotaxis contributed to floral diversity? In this review, I summarize recent progress in studies on two related fields to develop answers to these questions. First, it is known that molecular and cellular stochasticity are inevitably found in biological systems, including plant development. Organisms deal with molecular stochasticity in several ways, such as dampening noise through gene networks or maintaining function through cellular redundancy. Recent studies on molecular and cellular stochasticity suggest that stochasticity is not always detrimental to plants and that it is also essential in development. Second, studies on vegetative and inflorescence phyllotaxis have shown that plants often exhibit variability and flexibility in phenotypes. Three types of phyllotaxis variations are observed, namely, fluctuation around the mean, transition between regular patterns, and a transient irregular organ arrangement called permutation. Computer models have demonstrated that stochasticity in the phyllotactic pattern formation plays a role in pattern transitions and irregularities. Variations are also found in the number and positioning of floral organs, although it is not known whether such variations provide any functional advantages. Two ways of diversification may be involved in angiosperm floral evolution: precise regulation of organ position and identity that leads to further specialization of organs and organ redundancy that leads to flexibility in floral phyllotaxis.
\end{abstract}

Keywords Evo-Devo $\cdot$ Floral development $\cdot$ Floral phyllotaxis $\cdot$ Stochasticity $\cdot$ Variation

\section{Introduction}

Besides the remarkable diversity in form of angiosperm flowers, floral organ numbers and arrangements are relatively invariant, especially in core eudicots and monocots (Fig. 1). Floral phyllotaxis, the arrangement of floral organs, is largely classified into whorled (cyclic) and spiral. In contrast to core eudicots and monocots, which usually show whorled phyllotaxis, different angiosperm clades show wide floral phyllotaxis variation within and among species.

Miho Stephanie Kitazawa

kitazawa@celas.osaka-u.ac.jp

1 Center for Education in Liberal Arts and Sciences, Osaka University, 1-16 Machikaneyama-cho, Toyonaka, Osaka 560-0043, Japan
Solving the evolutionary history of floral phyllotaxis is not a simple problem, since both spiral and whorled phyllotaxis are observed in taxa in 'ANA' grade (Fig. 1a) that are sister to the major diversification of angiosperms (Smyth 2018). Recently, the ancestral floral phyllotaxis was reconstructed as whorled in the perianth and the androecium, and spiral in the gynoecium (Sauquet et al. 2017). This reconstructed ancestral flower has triggered arguments on the evolution of floral phyllotaxis, especially regarding its development (DePaula et al. 2018; Rümpler and Theißen 2019; Sokoloff et al. 2018). The development of phyllotaxis has been studied over a century, under both experimental and theoretical studies. Application of these theories to floral phyllotaxis will benefit studies on floral evolution.

Recently, studies focusing on phenotypic variation and developmental stochasticity have advanced rapidly, 
Fig. 1 Angiosperm phylogeny and floral ground plans. a Simplified angiosperms phylogeny (modified from Angiosperm Phylogeny Group 2016). Some branches are not presented since they are not mentioned in this paper. b Monocot and $\mathbf{c}$ eudicot floral ground plans, depicted by floral diagrams, which are widely used to discuss floral diversity (Ronse De Craene 2010)

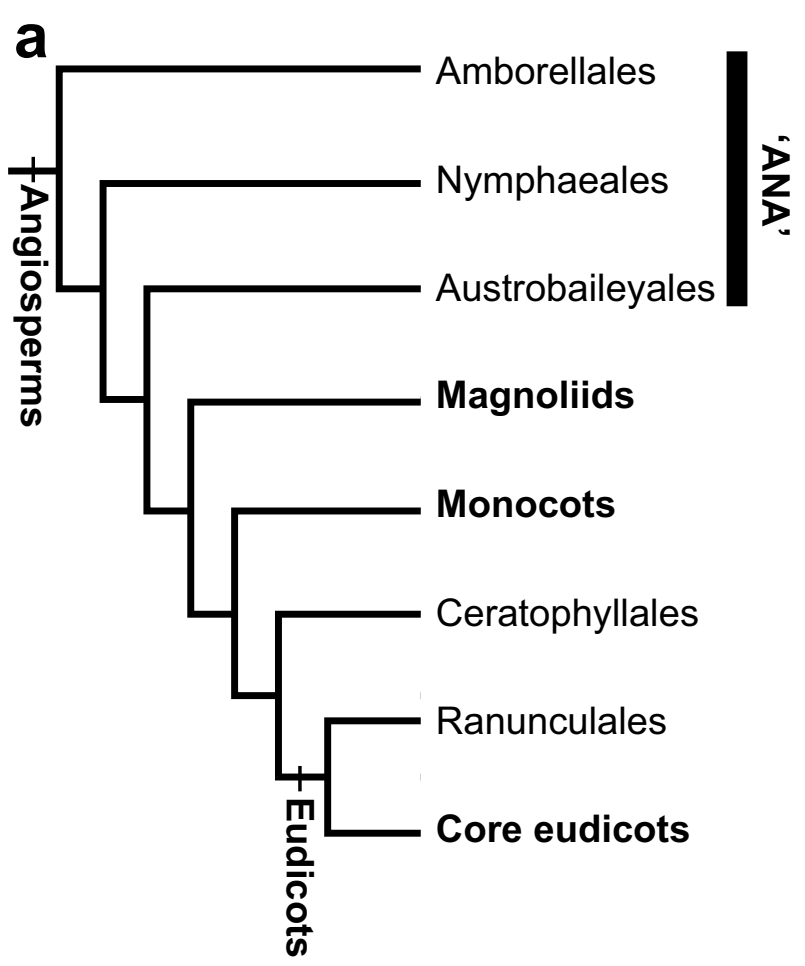

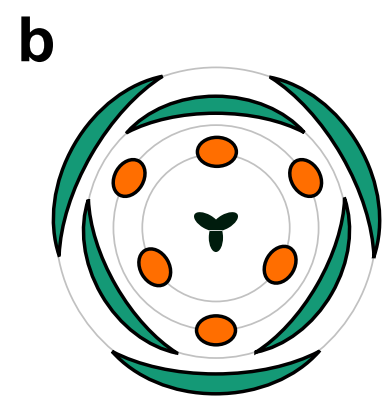

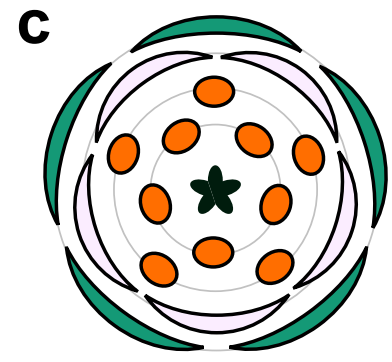

including the research fields related to phyllotaxis. Traditionally, the average, usually the mean but sometimes the mode, is considered, and variations are often ignored. Mean-centric approaches, however, lose a lot of information (Geiler-Samerotte et al. 2013). We have begun to understand that organisms can utilize stochasticity in various processes, such as organ development and cell-fate decisions (Meyer and Roeder 2014). As sessile organisms, plants have the ability to respond to fluctuating environments by changing their development, which results in varied and flexible architectures and phenotypes. For some traits, variations could be a result of adaptive evolution (Abley et al. 2016). In this review, I provide an overview of the molecular and cellular stochasticity in the developmental processes and instability of phyllotaxis, which is helpful in understanding floral organ positioning and how stochasticity contributes to floral development and evolution.

\section{Stochasticity in plant development}

Biological systems ubiquitously exhibit stochasticity in traits and processes, from the molecular to the multicellular level, even in developmental processes of robust and consistent phenotypes. Computational and experimental approaches have been used to study stochasticity in biological systems (Meyer and Roeder 2014). For example, a recent review on stochasticity in cellular fate decisions compared it to a weighted coin toss (Zechner et al. 2020). We have now begun to understand the mechanisms underlying the figurative coin toss model and to identify the factors contributing to the associated coin weights in various developmental processes.

\section{Robust development of multicellular tissue under molecular, cellular, and environmental stochasticity}

Cells in identical genetic and environmental backgrounds can show phenotypic diversity. It has been shown that gene expression shows stochasticity caused by intrinsic noise (fluctuations), produced by biochemical processes involved in gene expression, and extrinsic noise from other cellular components (Elowitz et al. 2002; Kærn et al. 2005; Raser and O'Shea 2005). In multicellular organisms, stochasticity in gene expression can be affected by multicellularity. For example, the stochasticity of the protein expression level among neighboring cells in young leaves is spatially correlated (Araújo et al. 2017). The correlation is not sufficiently explained by the inheritance of mRNA and proteins, suggesting that the inheritance of cellular condition and/or cell-cell communication is required (Araújo et al. 2017).

Variability in gene expression is not the only source of phenotypic variations. Examples from animals have shown that mechanical properties of cells and molecular fluctuations in the dynamics of signaling pathways affect cellular fate decision (Kroll et al. 2020; Weinreb et al. 2020; Zechner et al. 2020). Significant advancements 
have been made in research on stochasticity in cellular size and patterning (Meyer and Roeder 2014). Cellular sizes fluctuate around the mean established by the ploidy (Roeder et al. 2010), and the dynamics of endoreduplication that cause ploidy variation are identified as a stochastic Poisson process (Kawade and Tsukaya 2017). Spatial variation in the growth rate among neighboring cells is several folds in magnitude and sub-cellular differences in cell periphery have been observed (Elsner et al. 2012). Heterogeneity in turgor pressure correlating with cellular topology and geometry is observed in shoot apexes, although it is noted that heterogeneity may not always be stochastic (Long et al. 2020). Cell division orientation can be stochastic, indicated by a population of similarly shaped cells showing different division planes, contrary to the traditional idea that the division plane is uniquely determined by cellular shape (Besson and Dumais 2011). Moreover, plant aerial parts are exposed to fluctuating environments, and have response mechanisms to buffer these fluctuations (Walter and Schurr 2005). Such stochasticity in various aspects of cellular properties and environmental noises can affect multicellular development.

Several ways to control the noise, including molecular, cellular, or environmental noise, have been suggested, such as regulating the frequency of transcription and translation, controlling gene copy number, regulation by network motifs such as negative feedback, network redundancy, intercellular signaling, physical constraint, and mechanical feedback (Abley et al. 2016; Lachowiec et al. 2016; Lempe et al. 2013; Raj and van Oudenaarden 2008; Raser and O'Shea 2005). Local rules that describe interactions between components of systems, such as the connection of genes in gene regulatory networks or cell-cell communication in multicellular tissues, are often sufficient to form robust patterns (Long and Boudaoud 2019). Additionally, cellular or morphological redundancy can contribute to maintaining function where stochasticity in processes is affected. In vulval development of the nematode Caenorhabditis elegans, there are greater numbers of precursor cells than are actually required for functional vulvae, and only half of them adopt a vulval fate. Although the position of the cells adopting a vulval fate vary slightly among individuals, most of the non-canonical phenotypes are functional (Braendle and Félix 2008). This suggests that cellular redundancy plays a buffering role. Similarly, plants have morphological redundancy at a larger scale, such as repetitive organ (e.g., leaf) formation and reticulated vein networks that allow multiple alternative pathways for transportation of water and photosynthetic products (Lachowiec et al. 2016). Such redundancy can maintain functional robustness under fluctuations.
Utilizing stochasticity during plant development

Contrary to traditional views that considered variable phenotypes to be the result of poorly buffered development, recent studies suggest that it may be favored in some ecological scenarios, such as fluctuating and unpredictable environments (Abley et al. 2016; Kærn et al. 2005; Vogt 2015). Stochasticity is an important component prevalent in biological systems, including plant development, and stochastic developmental variation contributes to phenotypic diversity. Stochasticity can also play a constructive role in the development and cellular differentiation of multicellular organisms. Plants have systems that enhance cellular heterogeneity in growth (Uyttewaal et al. 2012) and it has been suggested that heterogeneity is required to produce specific tissue mechanical properties such as sepal curvature (Roeder et al. 2010, 2012) and stable organ shape (Hong et al. 2016). Stochasticity can be used to promote robustness through spatiotemporal averaging to maintain diversity in cell size in leaf epidermises and/or to create subtle differences among cells that initiate patterns by breaking symmetry (Long and Boudaoud 2019; Meyer and Roeder 2014; Roeder 2018). Stochasticity can cause phenotype shifts associated with network architecture such as the re-wiring of key-gene connections in response to changing environments (Lachowiec et al. 2016) and positive feedbacks that can result in switching between multiple states (Raj and van Oudenaarden 2008). The effect of noise can appear not only at the cellular level, but also at the tissue or organ level. For example, noise in the pattern formation process can alter the resulting organ position (Mirabet et al. 2012). These facts suggest that stochasticity plays an important role in plant development.

\section{Instability in phyllotaxis}

Phyllotaxis is the arrangement of repeated units, represented by leaves, around a plant stem (Fig. 2). Plants fall mostly into two categories: spiral phyllotaxis, where the divergence angle between successive two organs is constant, and whorled phyllotaxis, in which several organs occur at the same level on the stem. Both classes differ in their development. In spiral phyllotaxis, organ primordia arise sequentially in constant plastochrons (time-intervals) and constant divergence angles. Whereas in whorled phyllotaxis, several primordia comprising a whorl initiate simultaneously and equidistantly from the shoot apex. Spiral phyllotaxis with a divergence angle of approximately $137.5^{\circ}$ (the golden angle) is widely observed in the plant kingdom, from bryophytes to angiosperms, and even in distantly related taxa such as brown algae (Peaucelle and Couder, 2016).

The regularity of phyllotactic patterns has attracted the attention of many researchers over the centuries, with 
Fig. 2 Major phyllotactic patterns. a Whorled and $\mathbf{b}$ spiral phyllotaxis. In each column, the upper panel is the lateral view and the middle panel is the top view. In the bottom panels, positions of lateral organs are indicated by solid circles. Black lines in $\mathbf{a}$ indicate the whorls, whereas in $\mathbf{b}$ the black line indicates an ontogenic spiral

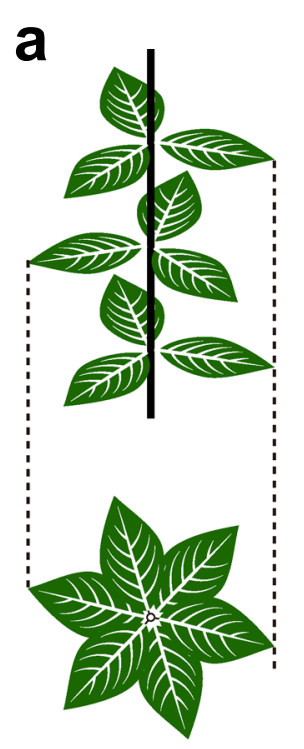

b
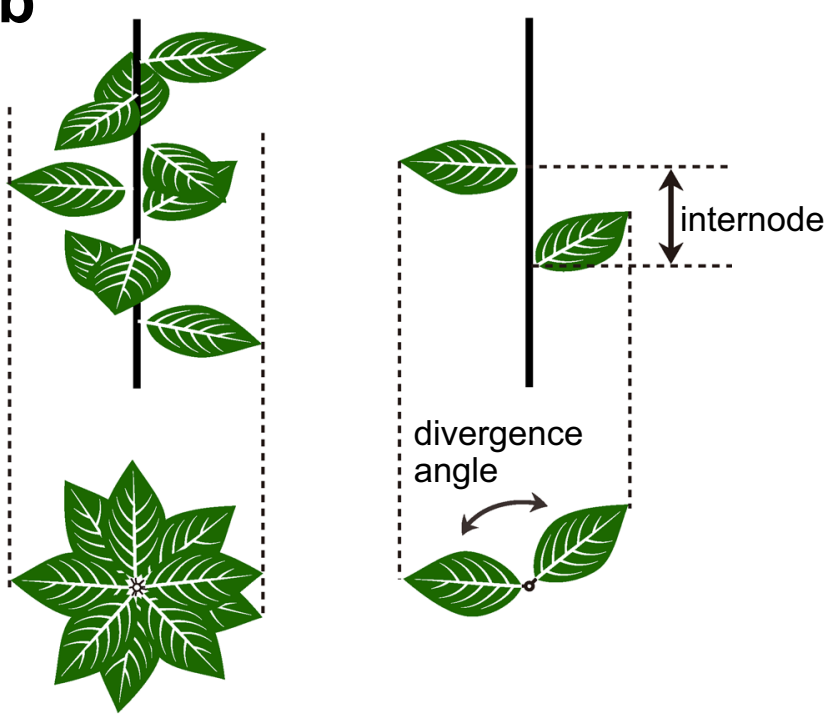

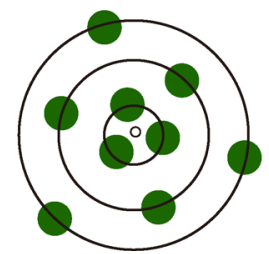

systematic studies beginning in the 1830s (Adler et al. 1997). Advantages of the common phyllotactic patterns in light capture and package efficiency have been discussed and several developmental mechanisms to achieve regularity have been suggested; for example, physical contact pressure among leaf primordia and chemical inhibitory fields. In angiosperms, lateral organ primordia are successively initiated at the shoot apex; therefore, phyllotactic patterns form around the shoot apical meristem (SAM). The central zone (CZ) of a SAM is an undifferentiated stem cell niche, and the peripheral zone (PZ) is the region where the cells undergo differentiation and where organ primordia are initiated. Theoretical studies have suggested the importance of SAM size on phyllotactic patterns. In Arabidopsis thaliana (L.) Heynh., CLAVATA-WUSCHEL (CLV-WUS) feedback signaling has been suggested as a central regulatory pathway for maintenance of the stem cell population in SAM (Brand et al. 2000; Schoof et al. 2000), and this regulatory pathway is likely conserved among angiosperms (Somssich et al. 2016). Feedback loop regulation enables the robust maintenance of the meristem size, even when the expression level of $C L V 3$ varies over a ten-fold range (Müller et al. 2006). Organ primordia initiation occurs in the PZ at the maxima of plant hormone auxin concentration. Auxin is transported by the efflux carrier PIN1 upward to the apex and accumulates, specifying the position of the primordium during early stages (Reinhardt et al. 2003). The expression

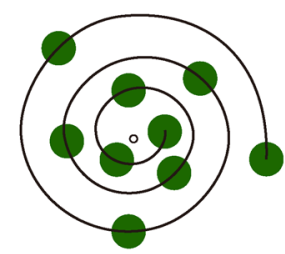

of auxin carriers suggests that the heterogeneous distribution at the surface layer is the main requirement for organ pattern formation. Computer models have shown that simple local mechanisms, where a difference in auxin concentration among the cells causes the polarization of auxin efflux carriers, are sufficient to generate common phyllotactic patterns, such as the golden spiral and decussate pattern (Jönsson et al. 2006; Smith et al. 2006).

\section{Variation in phyllotaxis: fluctuations, transition, and permutation}

Although it has been emphasized that the golden spiral and other regular patterns are widely observed in nature and many theoretical models succeeded to explain its stability, the observed occurrences of phyllotactic patterns are not invariant. Variation in phyllotaxis is often found in many species. There are roughly three types of variations: fluctuation around average, transition between multiple modes, and stochastic changes of the sequence, known as permutation.

Fluctuation around the average value has been observed in multiple species and mutants. For example, observations of mature Rhododendron branches showed that although the mean divergence angle is usually well-defined, individual divergence angles showed variations in branches (Bursill and Rouse 1998). In A. thaliana, divergence angles vary around the golden angle in wildtypes, and the degree of variation 
increases in some mutants. The variation in divergence angles and initiation timing can be generated around SAM; for example, stochasticity can exist in auxin dynamics or SAM and/or organ primordia size. In a quadruple mutant of auxin influx carriers, the divergent angle became irregular and PIN1 polar localization displayed a more diffuse pattern compared to the wild type, suggesting that rapid intake of auxin by influx carriers is required for stable regular patterning (Bainbridge et al. 2008). Mutants of genes regulating methyl esterification status of cell wall pectins show different degrees of variation in divergence angles around SAM compared to wildtypes, which can either be higher or lower depending on the mutated gene, suggesting that cell wall properties at primordium formation control the variability of primordium position (Peaucelle et al. 2011). Postmeristematic modification can also cause variations in the divergence angles and internode lengths on mature stem. In post-meristematic development, several genes, represented by CUP-SHAPED COTYLEDON (CUC) genes, are required to establish the boundary domain surrounding organ primordia. In plants expressing an miR164-resistant $C U C 2$ gene with a broader boundary domain, phyllotactic patterns are disrupted with a uniform distribution of divergence angles, in contrast to the maximum at $120^{\circ}-150^{\circ}$ in wildtype plants (Peaucelle et al. 2007). This irregularity is observed only in mature patterns and not in organ initiation patterns around SAMs (Burian et al. 2015; Peaucelle et al. 2007). The degree of variation is also affected by environmental conditions, such as day-length, associated with changes in SAM size (Landrein et al. 2015). Interestingly, the degree of variation is not the lowest in wildtypes in A. thaliana (Landrein et al. 2015; Peaucelle et al. 2011), suggesting a possibility that plants regulate it to keep a specific degree of variation.

In the theoretical models, multiple phyllotactic patterns including the golden spiral are generated and the transition between patterns occurs by changing phyllotactic parameters, such as size ratio between primordium and meristem. In nature, transitions between regular phyllotactic patterns are often observed. Transition between decussate to spiral phyllotaxis is commonly found in ontogeny of dicots, as they develop from two oppositely positioned cotyledons (Couder 1998; Meicenheimer 1998). Transitions can occur not only between spiral patterns but also between spiral and whorled phyllotaxis in the ontogeny where the area of the apical dome increases with time (Kwiatkowska and FlorekMarwitz 1999). Transitions are rather common when the developmental phase is shifted, for example from the vegetative to the reproductive phase, but it occurs even in the same phase generating primordia with the same identity (Kwiatkowska 1997; Kwiatkowska and Florek-Marwitz 1999). In the transient pattern between two regular patterns, different patterns or anomalous forms can appear, including oscillations in divergence angles (Smith et al. 2006) and an incompletely split leaf in the increased number of organs within a whorl in whorled phyllotaxis (Douady and Couder 1996c). The transitions are affected by how phyllotactic parameters change. For instance, types of transition changes depending on whether SAM size changes in a global and symmetrical manner or only in localized sectors (Meicenheimer and Zagorska-Marek 1989; Zagórska-Marek 1987). In mutants of model plants, non-random changes of phyllotactic patterns are observed. In maize, the mutant with the ABERRANT PHYLLOTAXY1 (ABPHI) gene, which encodes cytokinin response regulator, shows opposite (decussate) phyllotaxis with enlarged SAM, in contrast to the distichous phyllotaxis in wildtypes (Giulini et al. 2004; Jackson and Hake 1999). In A. thaliana, three members of PLETHORA (PLT)-like AP2 domain transcription factors redundantly control organ positioning. The triple mutant of PLT genes shows nonrandom change in phyllotaxis, where successive leaves can either be at $180^{\circ}$ or $90^{\circ}$ (Prasad et al. 2011). Although it is suggested that PLT proteins act as PIN1 regulators, the alternation of phyllotaxis in plt mutant is not directly caused by PIN1 level but by the accumulation of auxin at the center of SAM (Pinon et al. 2013). These studies suggest that meristem size as well as auxin dynamics play a central role in the transition between regular phyllotactic patterns.

Irregular sequences of organ arrangement or permutation are observed in many species (Barabé and Lacroix 2020; Refahi et al. 2016). A major example of permutation is an "M-shaped" motif appearing in spiral phyllotaxis of a divergence angle $\alpha^{\circ}$, showing a transient sequence of $2 \alpha^{\circ}, 360-\alpha^{\circ}$, $2 \alpha^{\circ}$ (Besnard et al. 2014; Couder 1998; Guédon et al. 2013). This motif is observed even in A. thaliana wildtypes, and appeared much more frequently in a mutant of cytokinin signaling inhibitor AHP6 (Besnard et al. 2014). This mutant shows a regular divergence angle and irregular plastochrons, and it is suggested that cytokinin regulates organ initiation after positioning by auxin, parallel with or downstream of auxin (Besnard et al. 2014). Studies on a mutant with less variability in divergence angle suggested that an enlarged meristem size promotes a shorter plastochrone, leading to an increase in the frequency of organ permutations (Landrein et al. 2015). It is likely that the permutation is not independent from the variation around the mean, and permutation may occur when the degree of variation exceeds a certain threshold (Barabé and Lacroix 2020).

\section{Abstract models of phyllotaxis and examination of the effect of noise}

The fundamental effect of noise has been examined using the abstract model of the phyllotaxis. The model framework established by Douady and Couder (1996a, b) is based on the idea that preexisting organs inhibit the initiation of new 
organ primordia. Douady and Couder's first model (Douady and Couder 1996a) assumed that the organ primordia are initiated one at a time, at a certain time interval, where the inhibitory energy exerted by preexisting primordia at the edge of the SAM, idealized as a disc, is at a minimum. The second model (Douady and Couder 1996b) discards the time-periodicity assumption and assumes one or more primordia initiate when the energy falls below a threshold value. The golden spiral patterns appeared in a wide range of parameter spaces of the models, suggesting robustness of the pattern to parameter fluctuations.

In phyllotactic pattern formation, there can be several types of noise due to different factors, such as the discrete nature of cellular tissue, inhibition strength depending on auxin level, sensitivity of cells to signals, and activity of the apex that would affect the effective radius of the generating circle and inhibitory range (Mirabet et al. 2012). Abstract models are utilized to study the effect of such noise, as the large number of parameters and details of the molecular mechanisms of realistic models may obscure the behavior of the system. At the same time, mapping between cell-based and abstract models has also been performed, enabling us to link molecular mechanisms and macroscopic behaviors of the system (Mirabet et al. 2012). Introducing noise to the abstract model reproduced some phyllotactic patterns observed in nature, for instance, a transient distichous pattern with $180^{\circ}$ and M-shaped sequences of angles (Mirabet et al. 2012). Another model assumed that primordium initiation is a stochastic process depending on the local level of inhibition, instead of the initiation threshold employed in the abstract model (Refahi et al. 2016). Simulations of stochastic models showed dynamic behaviors not explained by the deterministic model (Refahi et al. 2016).

\section{Floral phyllotaxis and development}

Flowers consist of several types of floral organs. Besides a few exceptions, floral organs are arranged in the order of carpels (female organs), stamens (male organs), and perianth organs, from inside to outside (Fig. 1b, c). As floral organs show spiral or whorled arrangement as leaves around a stem, it can be imagined that the organ pattern formation of floral phyllotaxis is similar to that of vegetative phyllotaxis. However, some processes in floral development are not common or not found in vegetative phyllotaxis.

\section{Developmental processes specific to flowers}

Where and when initiation of the floral organ primordia occurs play an important role in floral development. Numerous studies on floral ontogeny have shown that it is a dynamic and diversified process. As in the development of vegetative phyllotaxis, floral organs are initiated around a floral meristem, although there are many exceptions depending on species and/or developmental stages. Some specialized organ initiations are observed, such as the ring meristem, for example in Ranunculales (Becker 2016), a common primordium that further develops into multiple organ primordia, such as in Fabaceae (Tucker 2003), and early and late fusion of primordia, such as in Antirrhinum majus L. (Rebocho et al. 2017).

Flowers are a determinate shoot where the number of floral organs produced is usually programmed, contrary to the indeterminate growth commonly found in vegetative shoots. The organ initiation direction along with the inside-outside axis of a flower is diversified during floral evolution. The developing vegetative apex commonly shows centripetal development, where the outer organs are initiated first and the central organ forms last. In floral development, centrifugal development, where organs develop outwards from the center, is observed in addition to centripetal development (Rudall 2010). Two types of centrifugal development are recognized: intrazonal centrifugal development, in which the organ primordia within a zone (e.g., androecium zone) show centrifugal initiation, and interzonal centrifugal development, where an entire organ zone is initiated after the zone inside it, for example when stamen initiation is earlier than petal emergence (Rudall 2010). Bidirectional prepatterning occurs where the number and position of some organs (usually stamens) are dependent on positional information related to the inner organs (carpels) (Sokoloff et al. 2018). In contrast, unidirectional prepatterning relies on positional information from preexisting organs outside the organ-generating region in centripetal development, as in vegetative shoots (Sokoloff et al. 2018). The timing of termination of the central meristem or the timing of carpel formation can affect floral organ positioning and initiation order by limiting space and/or giving positional information from inner organs.

Floral organ primordia acquire a specific identity depending on their position in the floral bud, and floral organ identity can change the primordium size and cause transitions between phyllotactic patterns during floral ontogeny (Wiss and Zagórska-Marek 2012). The concept of floral organ fate determination was suggested to follow an $\mathrm{ABC}$ model (Fig. 3), based on homeotic mutants of floral organs in two core eudicot species, A. thaliana and A. majus (Bowman et al. 1991; Coen and Meyerowitz 1991). The original model suggested concentric expression of three classes of genes (named A, B, and C), which divide the floral bud into four concentric regions and specify floral organ identity. The expression of A-class genes specifies sepal fate, A- and B-class genes specify petal fate together, B- and C-class genes specify stamen fate together, and C-class genes specify carpel fate. A later study found that another class, named 
Fig. 3 Shoot structure of a flower and $\mathrm{ABCE}$ model of organ-fate determination. A flower is a shoot with a very short stem (dashed lines) terminating with gynoecium formation. Lateral organs in the flower, or floral organs, are differentiated into several types depending on the expression pattern of fate determinants

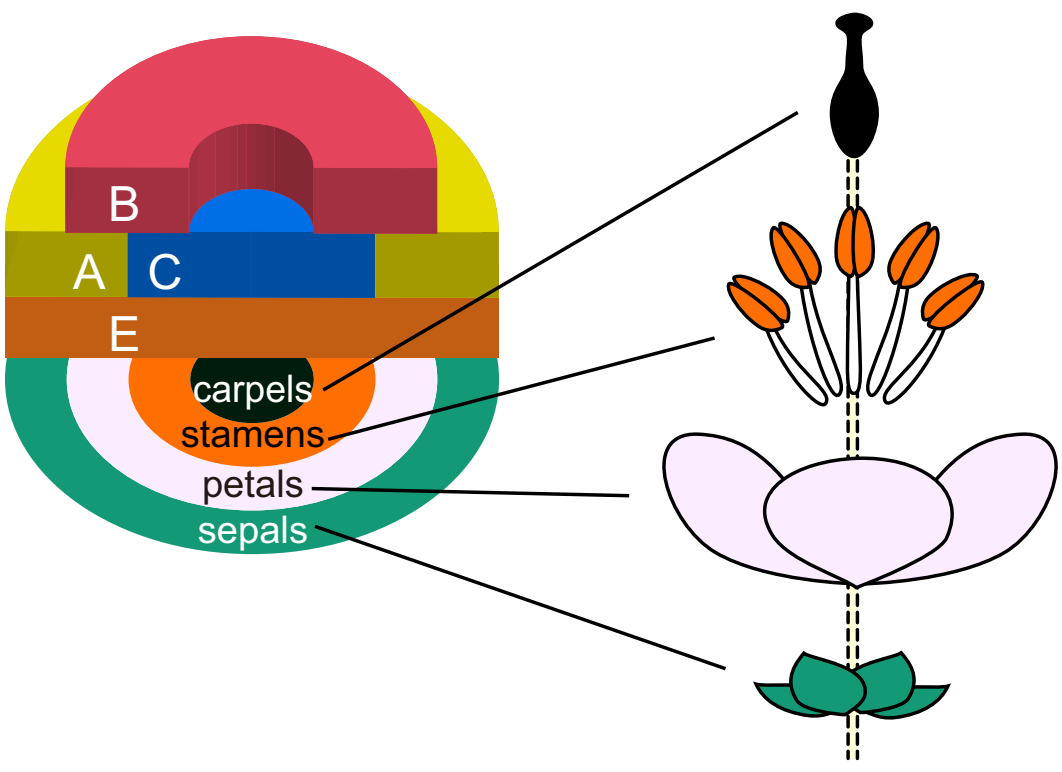

E, is required for floral organ identity (Fig. 3) (Pelaz et al. 2000). The modified ABC model has been applied to other species, covering a wide range of angiosperms. In non-grass monocots such as tulips, which have two morphologically similar outer whorls, expansion of B-class gene expression to the first whorl accounts for the two petaloid perianth whorls (Kanno et al. 2007). Such shifts in the boundary of expression domain, also known as the "sliding boundary model," can to some extent explain floral diversity (Bowman 1997; Kramer et al. 2003). Wider expressions of ABCE homologs, compared with the core eudicot model systems, are observed in other angiosperm clades such as magnoliids, Nymphaeales, Amborellales (Fig. 1a) (Chanderbali et al. 2010; Kim et al. 2005; Soltis et al. 2007; Zhang et al. 2020). The "fading borders" model has been suggested for these species, as there is a fading gradient of ABCE gene expressions observed between adjacent whorls (Buzgo et al. 2004; Soltis et al. 2007). The modified ABC model is considered to be universally applicable for organ specification in angiosperm flowers, providing a fundamental framework for understanding floral development and evolution. The differentiation itself is independent of organ positioning, but the specialized growth of each organ type can affect the initiation of subsequent organs.

Organ position can be modified by protruding position control after auxin patterning, post-meristematic regulations by boundary domain, pressure by close contact of organs, and limitation of space. The appearance of mature flowers and their development can differ, exemplified by rapid spiral initiation in calyx whorls in core eudicot species (Endress and Doyle 2007). Mathematical modelling studies have shown that the post-meristematic modification of an organ by repulsive interaction between organs enables concentric arrangement following spiral initiation and stabilizes the organ number in each circle (Kitazawa and Fujimoto 2015, 2020). This result suggests that interaction among organs, which is affected by the determinate nature of flowers and the growth control of each organ, can be crucial to ensure robust organ positioning.

\section{Variations in floral phyllotaxis}

Intraspecific variations in floral traits are well-known in some species (Fig. 4a). In the nineteenth century, Hugo de Vries performed a sequence of selection of Ranunculus bulbosus L. (Ranunculaceae) on the petal numbers and observed changes in variation curves (de Vries 1894). Aestivation variations in Ranunculus repens $\mathrm{L}$. was also reported in the mid-twentieth century (Cunnell 1958, 1964). Differences in organ initiation patterns among individuals have also been observed, for example, in Magnolia (Magnoliales) (Zagórska-Marek 1994), Ceratophyllum (Ceratophyllales) (Iwamoto et al. 2003), and Anemone (Ranunculales) (Ren et al. 2010). As in other biological processes, buffering systems for molecular stochasticity and the role of stochasticity have been suggested to influence floral development. Some features of three types of the variation, described above, are also observed in floral phyllotaxis.

Transition between regular patterns is observed in flowers, for example in Magnolia flowers (Wiss and ZagórskaMarek 2012; Zagórska-Marek 1994). Similar to vegetative phyllotaxis, the size ratio between floral meristems (FM) and floral organ primordia plays a central role in the determination of floral organ number and arrangement. In Magnolia flowers, the perianth is usually trimerous whorled and the androecium shows spiral patterns, and the relative size of organ primordia to FM size is smaller in the androecium than in the perianth (Wiss and Zagórska-Marek 2012). In 
a
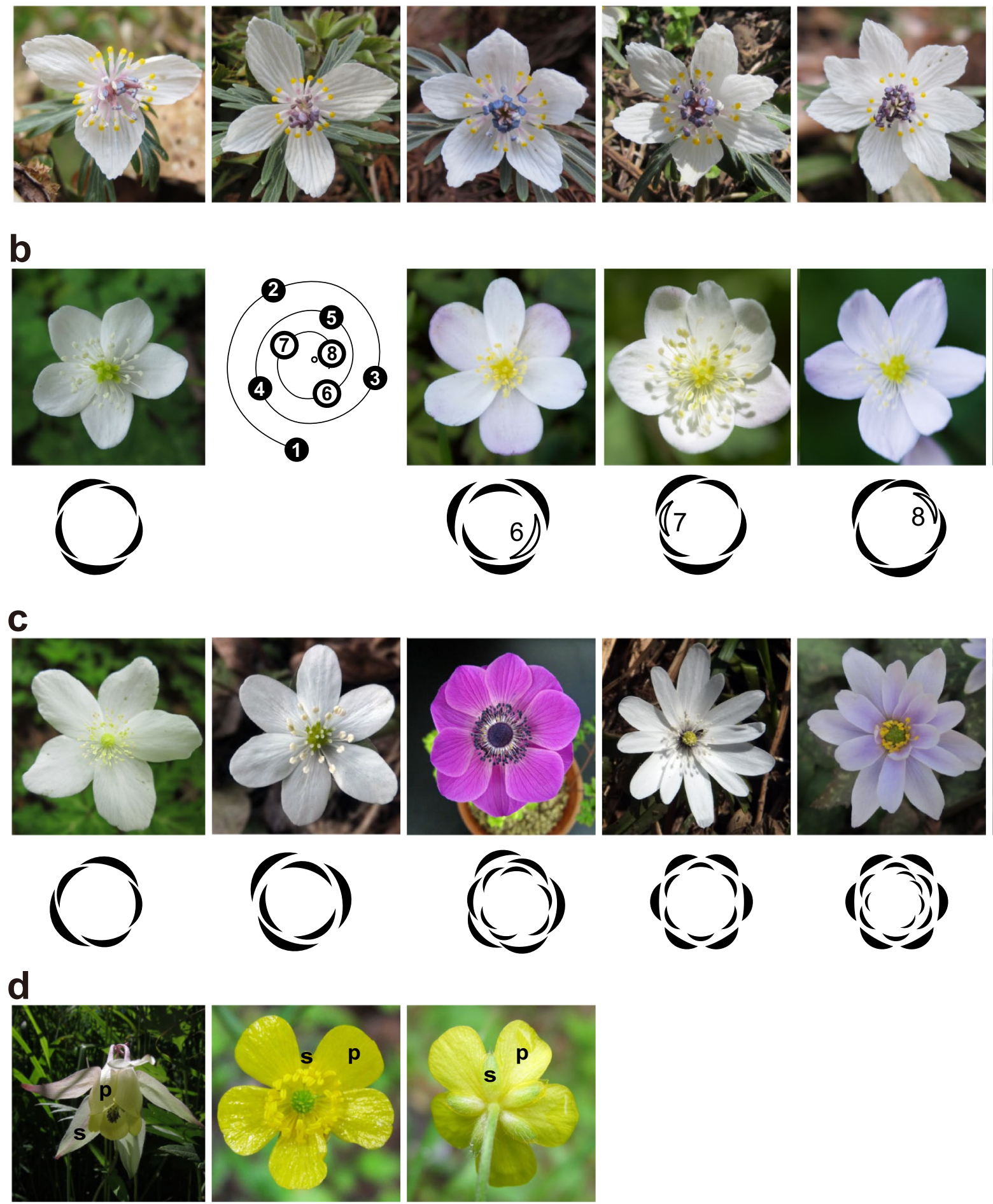

Linanthus, flowers with a higher number of corolla lobes showed larger FM diameters (Stevens et al. 1972). Mutations in the genes required for meristem maintenance, namely $W U S$ and $C L V$, show altered apical meristems not only in SAMs but also in FMs, with abnormal organ numbers and larger variations in organ positions (Clark et al. 1993, 1995;
Laux et al. 1996; Schoof et al. 2000). Similar to the transient process in vegetative development, a transient deviation from normal patterns can appear between regular patterns. For example, divergence angles in early floral development oscillatory approaches $144^{\circ}$, equidistant angular placing of five organs in pentamerous flowers (Lyndon 1978). 
४Fig. 4 Variation and diversity in Ranunculaceae flowers. a Variation of sepal numbers in Eranthis pinnatifida Maxim. b Typical flower of Anemone flaccida F.Schmidt with five tepals (left) and flowers with six tepals with different arrangements (three panels on the right). Numbers in the schematic diagram show the initiation order of floral organs in a spiral phyllotaxis. Tepal arrangements are shown in floral diagrams below the photographs. If the sixth tepal is in the position predicted by spiral phyllotaxis, tepal arrangement must have three outer tepals and three inner tepals (middle panel). However, two other arrangements were observed, indicating that the sixth tepal is not limited to the position predicted by spiral phyllotaxis (two panels on the right). c Anemone flowers, ordered by tepal numbers. From left to right, A. nikoensis Maxim., A. hepatica var. japonica (Nakai) Ohwi, A. coronaria L., A. raddeana Regel, and A. keiskeana T.Itô ex Maxim. Floral diagrams show the arrangement of tepals in the photographs. d Flowers of Aquilegia buergeriana Siebold et Zucc. (left) and Ranunculus sp. (middle and right). In contrast to Anemone flowers, perianth organs are differentiated into sepals ( $\mathrm{s}$ ) and petals (p)

Fluctuations in organ position can be caused by nongenetic factors, environmental conditions, stochastic events, and physical factors, such as external and internal pressures, and a perspective regarding flowers as dynamic random systems is being developed (Ronse De Craene 2018). Floral phyllotaxis can be altered by environmental factors, such as temperature (Crozier and Thomas 1993; Lyndon 1998). Not only the environment outside the plant body, but also the environment induced by other organs of the plant, can affect the floral development. For instance, in some mutants, the alternation of floral organ arrangements is associated with changes in the number and position of the surrounding bracts (Hepworth et al. 2005; Khan et al. 2014; Lee et al. 2007; Norberg et al. 2005). Furthermore, a correlation of floral construct with inflorescence has been observed, such as in Adoxa moschatellina L. with four petals in the terminal flower and five petals in the lateral flowers (Whitehead 1902). The difference between the terminal and lateral flowers can be a physical constraint by outer organs, which can change the geometry of floral meristem and lead to irregular patterns of subsequent organs (Rutishauser 2016). The expression patterns of genes can be different between the terminal and lateral flowers, depending on distinct genetic regulation between the terminal and lateral flowers (Rudall and Bateman 2003), or whether they have an inflorescence meristem to provide positional information. Such positional information, either physical or chemical, can cause differences in floral organ positioning (Nakagawa et al. 2020). A variation in floral phyllotaxis can be generated not only in the initiation of primordia but also after the initiation. For example, occasional splitting of primordia has been proposed as the mechanism underlying developmental variations in Microseris (Asteraceae) pappus part number, based on their Poissonian distribution (Bachmann and Chambers 1978).

In some plants with variable floral organ arrangement, exemplified by the family Ranunculaceae, the importance of organ identity determination has been emphasized as a source of variation. Floral organ primordia with different identities grow at different rates and reach different sizes; sepal primordia usually grow rapidly to cover the floral bud, in contrast to the petal primordia whose growth often occurs in the later stages of floral development. As the arrangements of previously formed organs can affect the phyllotaxis and stability of patterns of the subsequent organs (Endress 1989; Wiss and Zagórska-Marek 2012), the fluctuation in the fate determinant can cause variation in organ positioning in later developmental stages. In Nigella (Ranunculaceae) flowers, spiral arrangement of organ primordia has been suggested as a plausible source of the variation in organ number and arrangement (Wang et al. 2015). In spiral flowers, minor fluctuations in the expression boundary of identity determinants can be easily reflected in organ number, and this is consistent with predictions from organ number distributions (Kitazawa and Fujimoto 2014). Therefore, there is stochasticity in the expression border of organ identity genes, which can be amplified in non-whorled arrangements. Conversely, observations of mature Anemone flowers revealed that an occasional increase in outer organs (tepals) does not always follow the normal spiral of five tepals (Fig. 4b) (Kitazawa and Fujimoto 2016). It can be recognized as a stochastic "permutation," as the seventh or eighth primordium acquires an outer organ fate instead of the sixth primordium. However, it is not clear whether it is a permutation in the organ initiation or fate-determination. The former possibility could be supported by variations in primordium positioning observed in Ranunculaceae species (Ren et al. 2010, 2011). The latter corresponds to a possibility that the stochasticity in the position of extra tepals may be caused by the stochastic organ fate determination depending on the expression level of fate determinants, as in stochastic initiation of organ primordia depending on inhibitory fields (Refahi et al. 2016). This could explain how variations are generated in flowers with "fading borders" of fate determinants. Although the experimental evidence for fading borders is very weak except for in the angiosperm clades that are most distant to core eudicots (Becker 2016; Voelckel et al. 2010), analogous mechanisms can exist in flowers with considerable variation in organ number and arrangement.

\section{Floral evo-devo and stochasticity}

The number and arrangement of floral organs has played a major role in plant systematics, exemplified by Linnæus, who used stamen and carpel numbers for his taxonomy (Linnæus 1735). Although Linnæus's taxonomy ordering plant families by stamen and carpel numbers is rejected in modern biology, it is accepted that floral organ number and arrangement are largely associated with angiosperm phylogeny. A 
difference in merosity, the number of organs in each whorl, is known between eudicots and the other two major clades, monocots and magnoliids (Ronse De Craene 2010; Smyth 2018). The monocot ground plan comprises two trimerous whorls of undifferentiated perianth organs, two trimerous whorls of stamens, and three fused carpels (Fig. 1b). The ground plan of magnoliids is also regarded as trimerous, but the number of whorls is higher than that of monocots (Smyth 2018). The ground plan of core eudicot (or Pentapetalae [Cantino et al. 2007]) flowers is pentamerous whorled with two perianth whorls and two stamen whorls, with the two perianth whorls morphologically differentiated into calyx (sepal whorl) and corolla (petal whorl), with alternation of organs in adjacent whorls (Fig. 1c) (Chanderbali et al. 2017; Smyth 2018). Different merosities, especially tetramery, are often found in core eudicots. In addition to these groups with relatively stable floral construction, diversity and flexibility of the floral phyllotaxis among closely related species, or within a species, is present in 'ANA' grade, magnoliids, and non-core eudicot clades such as Ranunculales (Damerval and Becker 2017; Endress 1990).

The function of stochasticity in floral development has been investigated using a stochastic model of the floral organ identity regulatory network (Alvarez-Buylla et al. 2008). In the model, the attractors of Boolean networks of 15 genes corresponding to four floral organ identities were approximated. Owing to the deterministic nature of the Boolean gene regulatory network, transitions between fates cannot occur. Incorporating noise into the model enabled transitioning between the attractors. Interestingly, fate transition proceeds in the order of sepal, petal, carpel, and stamen, mimicking the order of floral development in a range of noise levels (0.5-10\%), and differences between the developmental timing of carpel and stamen diminish as the magnitude of noise increases. Thus, analysis of this model suggests that noise is required for cellular state transitions in floral development, and that stochasticity plays an important role, as in other biological systems.

Although stochasticity exists ubiquitously in the developmental process, the degree of noise required to change the morphology is different among species and organs. Genetic basis of robust ranges against perturbations has been evaluated in studies on the Brassicaceae species Cardamine hirsuta L. (Monniaux et al. 2016, 2018; Pieper et al. 2016). Most of the Brassicaceae species, including $A$. thaliana, show a stable floral construct with four sepals and four petals. On the contrary, $C$. hirsuta shows variations in petal numbers, ranging between zero and four. The variation depends on environmental perturbations such as day length and temperature, and is a heritable phenotype (McKim et al. 2017; Monniaux et al. 2016; Pieper et al. 2016). APETALA1 (API), which establishes floral meristem identity and acts as one of the A-class genes in Brassicaceae (Litt 2007), has been shown to be involved in the difference in the petal number variation between $C$. hirsuta and $A$. thaliana (Monniaux et al. 2018). These studies suggest a genetic basis of noise buffering in Brassicaceae species with stable petal numbers, and evolutionary change in the floral stability of $C$. hirsuta by releasing cryptic variation (Monniaux et al. 2018).

It is still uncertain whether intraspecific variations in floral phyllotaxis in extant species have any function, as in molecular or cellular stochasticity. It might merely be the result of poorly buffered developmental conditions, or advantageous, as is the case in phenotypic variations in microorganisms. Studies on stochasticity in development suggest two strategies to deal with molecular or cellular stochasticity, which may or may not involve floral diversification. The first strategy is the precise regulation of organ position and gene expression, where noise is buffered out. This strategy results in a stable floral construct, and diversification occurs through further specialization and close spatial and functional association of organs, known as synorganization (Endress 1990; Endress and Doyle 2007). The second strategy is organ redundancy, which allows the maintenance of function even when the organ fate changes, as in the cellular redundancy in the vulval development of $C$. elegans (Braendle and Félix 2008). Furthermore, this can be a driving force of the floral phyllotaxis diversity among closely related species, as in Ranunculaceae (Fig. 4c, d).

Inference of the ancestral flower of extant angiosperms triggered an argument on the possible combination of floral phyllotaxis in a flower (De-Paula et al. 2018; Rümpler and Theißen 2019; Sauquet et al. 2017; Sokoloff et al. 2018). Sauquet et al. (2018) argued that the transitions of phyllotaxis in different floral organ types may be caused by gradual changes in phyllotactic parameters, such as the size ratio between meristem and organ primordium; computer models will be helpful in examining possible transitions (Douady and Couder 1996c; Zagórska-Marek and Szpak 2008). Another perspective, not considered in the approach of Sauquet et al. (2017), is the coexistence of multiple states and the possibility that the ancestral state is polymorphic (Sauquet et al. 2018). Recently, we reported the coexistence of spirals and whorls in the perianth phyllotaxis at maturity in Anemone and Eranthis (Ranunculaceae) flowers (Kitazawa and Fujimoto 2020). Studies on phyllotaxis variations suggest that such variations are common in nature, and variable phenotypes can be generated from a single developmental system, with inevitable existence of stochasticity in biological processes.

\section{Conclusion}

Recent progress in molecular and cellular stochasticity suggested that plants utilize stochasticity in development. Although several phyllotactic patterns represented by the 
golden spiral appear robust, several types of variations are observed in nature, and the developmental sources of variations are discussed using mathematical models. In both vegetative and floral phyllotaxis, awareness about the importance of stochasticity has been increasing. Further clarification of the role of stochasticity in floral development will reveal new perspectives regarding the diversity of angiosperm flowers.

Acknowledgements I would like to thank Dr. Koichi Fujimoto and members of the Laboratory of Theoretical Biology, Osaka University, for valuable discussions and cooperation for the field work. I would also like to acknowledge anonymous reviewers who helped tremendously to improve the manuscript with constructive suggestions.

Funding No funding was received to assist with the preparation of this manuscript.

\section{Declarations}

Conflict of interests The authors have no relevant financial or nonfinancial interests to disclose.

Open Access This article is licensed under a Creative Commons Attribution 4.0 International License, which permits use, sharing, adaptation, distribution and reproduction in any medium or format, as long as you give appropriate credit to the original author(s) and the source, provide a link to the Creative Commons licence, and indicate if changes were made. The images or other third party material in this article are included in the article's Creative Commons licence, unless indicated otherwise in a credit line to the material. If material is not included in the article's Creative Commons licence and your intended use is not permitted by statutory regulation or exceeds the permitted use, you will need to obtain permission directly from the copyright holder. To view a copy of this licence, visit http://creativecommons.org/licenses/by/4.0/.

\section{References}

Abley K, Locke JCW, Leyser HMO (2016) Developmental mechanisms underlying variable, invariant and plastic phenotypes. Ann Bot 117:733-748. https://doi.org/10.1093/aob/mcw016

Adler I, Barabe D, Jean RV (1997) A history of the study of phyllotaxis. Ann Bot 80:231-244. https://doi.org/10.1006/anbo.1997. 0422

Alvarez-Buylla ER, Chaos A, Aldana M et al (2008) Floral morphogenesis: stochastic explorations of a gene network epigenetic landscape. PLoS ONE 3:e3626. https://doi.org/10.1371/journal. pone. 0003626

Angiosperm Phylogeny Group (2016) An update of the angiosperm phylogeny group classification for the orders and families of flowering plants: APG IV. Bot J Linn Soc 181:1-20. https://doi. org/10.1111/boj.12385

Araújo IS, Pietsch JM, Keizer EM et al (2017) Stochastic gene expression in Arabidopsis thaliana. Nat Commun 8:2132. https://doi. org/10.1038/s41467-017-02285-7

Bachmann K, Chambers KL (1978) Pappus part number in annual species of Microseris (Compositae, Cichoriaceae). Plant Syst Evol 129:119-134. https://doi.org/10.1007/BF00988986
Bainbridge K, Guyomarch S, Bayer E et al (2008) Auxin influx carriers stabilize phyllotactic patterning. Genes Dev 22:810-823. https:// doi.org/10.1101/gad.462608

Barabé D, Lacroix C (2020) Phyllotactic patterns: a multidisciplinary approach. World Scientific, Berlin

Becker A (2016) Tinkering with transcription factor networks for developmental robustness of Ranunculales flowers. Ann Bot 117:845-858. https://doi.org/10.1093/aob/mcw037

Besnard F, Refahi Y, Morin V et al (2014) Cytokinin signalling inhibitory fields provide robustness to phyllotaxis. Nature 505:417442. https://doi.org/10.1038/nature12791

Besson S, Dumais J (2011) Universal rule for the symmetric division of plant cells. Proc Natl Acad Sci 108:6294-6299. https://doi. org/10.1073/pnas.1011866108

Bowman JL (1997) Evolutionary conservation of angiosperm flower development at the molecular and genetic levels. J Biosci 22:515-527. https://doi.org/10.1007/BF02703197

Bowman JL, Smyth DR, Meyerowitz EM (1991) Genetic interactions among floral homeotic genes of Arabidopsis. Development 112:1-20

Braendle C, Félix M-A (2008) Plasticity and errors of a robust developmental system in different environments. Dev Cell 15:714-724. https://doi.org/10.1016/j.devcel.2008.09.011

Brand U, Fletcher JC, Hobe M et al (2000) Dependence of stem cell fate in Arabidopsis on a feedback loop regulated by CLV3 activity. Science 289:617-619. https://doi.org/10.1126/science.289. 5479.617

Burian A, Raczyńska-Szajgin M, Borowska-Wykręt D et al (2015) The CUP-SHAPED COTYLEDON2 and 3 genes have a postmeristematic effect on Arabidopsis thaliana phyllotaxis. Ann Bot 115:807-820. https://doi.org/10.1093/aob/mcv013

Bursill LA, Rouse JL (1998) Investigation of phyllotaxis of Rhododendron. In: Jean RV, Barabé D (eds) Symmetry in plants. World Scientific, Berlin, pp 3-32. https://doi.org/10.1142/9789814261 074_0006

Buzgo M, Soltis PS, Soltis DE (2004) Floral Developmental Morphology of Amborella trichopoda (Amborellaceae). Int J Plant Sci 165:925-947. https://doi.org/10.1086/424024

Cantino PD, Doyle JA, Graham SW et al (2007) Towards a phylogenetic nomenclature of Tracheophyta. Taxon 56:822-846. https:// doi.org/10.2307/25065864

Chanderbali AS, Yoo M-J, Zahn LM et al (2010) Conservation and canalization of gene expression during angiosperm diversification accompany the origin and evolution of the flower. Proc Natl Acad Sci 107:22570-22575. https://doi.org/10.1073/pnas.10133 95108

Chanderbali AS, Berger BA, Howarth DG et al (2017) Evolution of floral diversity: genomics, genes and gamma. Philos Trans R Soc Lond B Biol Sci 372:20150509. https://doi.org/10.1098/rstb. 2015.0509

Clark SE, Running MP, Meyerowitz EM (1993) CLAVATA1, a regulator of meristem and flower development in Arabidopsis. Development 119:397-418

Clark SE, Running MP, Meyerowitz EM (1995) CLAVATA3 is a specific regulator of shoot and floral meristem development affecting the same processes as CLAVATA1. Development 121:2057-2067

Coen ES, Meyerowitz EM (1991) The war of the whorls: genetic interactions controlling flower development. Nature 353:3137. https://doi.org/10.1038/353031a0

Couder Y (1998) Initial transitions, order and disorder in phyllotactic patterns: the ontogeny of Helianthus annuus: a case study. Acta Soc Bot Pol 67:129-150. https://doi.org/10.5586/asbp.1998.016

Crozier TS, Thomas JF (1993) Normal floral ontogeny and cool temperature-induced aberrant floral development in Glycine max (Fabaceae). Am J Bot 80:429-448. https://doi.org/10.2307/ 2445390 
Cunnell GJ (1958) Aestivation in Ranunculus repens L. New Phytol 57:340-352

Cunnell GJ (1964) Further observations on aestivation in Ranunculus repens L. New Phytol 63:1-11

Damerval C, Becker A (2017) Genetics of flower development in Ranunculales-a new, basal eudicot model order for studying flower evolution. New Phytol 216:361-366. https://doi.org/10. $1111 /$ nph.14401

de Vries H (1894) Ueber halbe Galton-Curven als Zeichen discontinuirlicher Variation. Ber Dtsch Bot Ges 12:197-207

De-Paula OC, Assis LCS, Ronse de Craene LP (2018) Unbuttoning the ancestral flower of angiosperms. Trends Plant Sci 23:551-554

Douady S, Couder Y (1996a) Phyllotaxis as a dynamical self organizing process part I: the spiral modes resulting from time-periodic iterations. J Theor Biol 178:255-273. https://doi.org/10.1006/ jtbi.1996.0024

Douady S, Couder Y (1996b) Phyllotaxis as a dynamical self organizing process part II: the spontaneous formation of a periodicity and the coexistence of spiral and Whorled patterns. J Theor Biol 178:275-294. https://doi.org/10.1006/jtbi.1996.0025

Douady S, Couder Y (1996c) Phyllotaxis as a dynamical self organizing process part III: the simulation of the transient regimes of Ontogeny. J Theor Biol 178:295-312. https://doi.org/10.1006/ jtbi.1996.0026

Elowitz MB, Levine AJ, Siggia ED, Swain PS (2002) Stochastic gene expression in a single cell. Science 297:1183-1186. https://doi. org/10.1126/science.1070919

Elsner J, Michalski M, Kwiatkowska D (2012) Spatiotemporal variation of leaf epidermal cell growth: a quantitative analysis of Arabidopsis thaliana wild-type and triple cyclinD3 mutant plants. Ann Bot 109:897-910. https://doi.org/10.1093/aob/ $\operatorname{mcs} 005$

Endress PK (1989) Chaotic floral phyllotaxis and reduced perianth in Achlys (Berberidaceae). Bot Acta 102:159-163. https://doi. org/10.1111/j.1438-8677.1989.tb00085.x

Endress PK (1990) Patterns of floral construction in ontogeny and phylogeny. Biol J Linn Soc 39:153-175

Endress PK, Doyle JA (2007) Floral phyllotaxis in basal angiosperms: development and evolution. Curr Opin Plant Biol 10:52-57. https://doi.org/10.1016/j.pbi.2006.11.007

Geiler-Samerotte KA, Bauer CR, Li S et al (2013) The details in the distributions: why and how to study phenotypic variability. Curr Opin Biotechnol 24:752-759. https://doi.org/10.1016/j. copbio.2013.03.010

Giulini A, Wang J, Jackson D (2004) Control of phyllotaxy by the cytokinin-inducible response regulator homologue ABPHYL1. Nature 430(7003):1031-1034. https://doi.org/10.1038/natur e02778

Guédon Y, Refahi Y, Besnard F et al (2013) Pattern identification and characterization reveal permutations of organs as a key genetically controlled property of post-meristematic phyllotaxis. J Theor Biol 338:94-110. https://doi.org/10.1016/j.jtbi. 2013.07.026

Hepworth SR, Zhang Y, McKim S, Li X, Haughn GW (2005) BLADE-ON-PETIOLE-dependent signaling controls leaf and floral patterning in Arabidopsis. Plant Cell 17:1434-1448. https://doi.org/10.1105/tpc.104.030536

Hong L, Dumond M, Tsugawa S et al (2016) Variable cell growth yields reproducible organ development through spatiotemporal averaging. Dev Cell 38:15-32. https://doi.org/10.1016/j.devcel. 2016.06.016

Iwamoto A, Shimizu A, Ohba H (2003) Floral development and phyllotactic variation in Ceratophyllum demersum (Ceratophyllaceae). Am J Bot 90:1124-1130. https://doi.org/10.3732/ajb. 90.8 .1124
Jackson D, Hake S (1999) Control of phyllotaxy in maize by the abphyl1 gene. Development 126:315-323

Jönsson H, Heisler MG, Shapiro BE et al (2006) An auxin-driven polarized transport model for phyllotaxis. Proc Natl Acad Sci 103:1633-1638. https://doi.org/10.1073/pnas.0509839103

Kærn M, Elston TC, Blake WJ, Collins JJ (2005) Stochasticity in gene expression: from theories to phenotypes. Nat Rev Genet 6:451-464. https://doi.org/10.1038/nrg1615

Kanno A, Nakada M, Akita Y, Hirai M (2007) Class B gene expression and the modified ABC model in nongrass monocots. Sci World J 7:268-279

Kawade K, Tsukaya H (2017) Probing the stochastic property of endoreduplication in cell size determination of Arabidopsis thaliana leaf epidermal tissue. PLoS ONE 12:e0185050. https://doi.org/10.1371/journal.pone.0185050

Khan M, Xu H, Hepworth SR (2014) BLADE-ON-PETIOLE genes: setting boundaries in development and defense. Plant Sci 215:157-171. https://doi.org/10.1016/j.plantsci.2013.10.019

Kim S, Koh J, Yoo M-J et al (2005) Expression of floral MADS-box genes in basal angiosperms: implications for the evolution of floral regulators. Plant J 43:724-744. https://doi.org/10.1111/j. 1365-313X.2005.02487.x

Kitazawa MS, Fujimoto K (2014) A developmental basis for stochasticity in floral organ numbers. Front Plant Sci 5:545. https:// doi.org/10.3389/fpls.2014.00545

Kitazawa MS, Fujimoto K (2015) A dynamical phyllotaxis model to determine floral organ number. PLoS Comput Biol 11:e1004145. https://doi.org/10.1371/journal.pcbi.1004145

Kitazawa MS, Fujimoto K (2016) Stochastic occurrence of trimery from pentamery in floral phyllotaxis of Anemone (Ranunculaceae). Acta Soc Bot Pol 85:3530. https://doi.org/10.5586/asbp. 3530

Kitazawa MS, Fujimoto K (2020) Perianth phyllotaxis is polymorphic in the basal eudicot Anemone and Eranthis species. Front Ecol Evol 8:70. https://doi.org/10.3389/fevo.2020.00070

Kramer EM, Di Stilio VS, Schlüter PM (2003) Complex patterns of gene duplication in the APETALA3 and PISTILLATA lineages of the Ranunculaceae. Int J Plant Sci 164:1-11. https://doi.org/ $10.1086 / 344694$

Kroll JR, Tsiaxiras J, van Zon JS (2020) Variability in $\beta$-catenin pulse dynamics in a stochastic cell fate decision in C. elegans. Dev Biol 461:110-123. https://doi.org/10.1016/j.ydbio.2020.02.004

Kwiatkowska D (1997) Intraspecific variation of phyllotaxis stability in Anagallis arvensis. Acta Soc Bot Pol 66:259-271. https://doi. org/10.5586/asbp.1997.031

Kwiatkowska D, Florek-Marwitz J (1999) Ontogenetic variation of phyllotaxis and apex geometry in vegetative shoots of Sedum maximum (L.) Hoffm. Acta Soc Bot Pol 68:85-95. https://doi. org/10.5586/asbp.1999.013

Lachowiec J, Queitsch C, Kliebenstein DJ (2016) Molecular mechanisms governing differential robustness of development and environmental responses in plants. Ann Bot 117:795-809. https://doi. org/10.1093/aob/mcv151

Landrein B, Refahi Y, Besnard F et al (2015) Meristem size contributes to the robustness of phyllotaxis in Arabidopsis. J Exp Bot 66:1317-1324. https://doi.org/10.1093/jxb/eru482

Laux T, Mayer KF, Berger J, Jürgens G (1996) The WUSCHEL gene is required for shoot and floral meristem integrity in Arabidopsis. Development 122:87-96

Lee DY, Lee J, Moon S, Park SY, An G (2007) The rice heterochronic gene SUPERNUMERARY BRACT regulates the transition from spikelet meristem to floral meristem. Plant J 49:64-78. https:// doi.org/10.1111/j.1365-313X.2006.02941.x 
Lempe J, Lachowiec J, Sullivan AM, Queitsch C (2013) Molecular mechanisms of robustness in plants. Curr Opin Plant Biol 16:6269. https://doi.org/10.1016/j.pbi.2012.12.002

Linnæus C (1735) Systema naturæ, sive, regna tria naturæ systematice proposita per classes, ordines, genera, \& species. In: Apud Theodorum Haak: ex typographia Joannis Wilhelmi de Groot, Leiden. https://doi.org/10.5962/bhl.title.877

Litt A (2007) An evaluation of A-function: evidence from the APETALA1 and APETALA2 gene lineages. Int J Plant Sci 168:73-91. https://doi.org/10.1086/509662

Long Y, Boudaoud A (2019) Emergence of robust patterns from local rules during plant development. Curr Opin Plant Biol 47:127137. https://doi.org/10.1016/j.pbi.2018.11.002

Long Y, Cheddadi I, Mosca G et al (2020) Cellular heterogeneity in pressure and growth emerges from tissue topology and geometry. Curr Biol 30:1504-1516. https://doi.org/10.1016/j.cub.2020.02. 027

Lyndon RF (1978) Phyllotaxis and the initiation of primordia during flower development in Silene. Ann Bot 42:1349-1360

Lyndon RF (1998) Phyllotaxis in flowers and in flower reversion. In: Jean RV, Barabé D (eds) Symmetry in plants. World Scientific, pp 109-124. https://doi.org/10.1142/9789814261074_0005

McKim SM, Routier-Kierzkowska A-L, Monniaux M et al (2017) Seasonal regulation of petal number. Plant Physiol 175:00563. https://doi.org/10.1104/pp.17.00563

Meicenheimer RD (1998) Decussate to spiral transitions in phyllotaxis. In: Jean RV, Barabé D (eds) Symmetry in plants. World Scientific, pp 125-143. https://doi.org/10.1142/9789814261074_0006

Meicenheimer RD, Zagorska-Marek B (1989) Consideration of the geometry of the phyliotaxic triangular unit and discontinuous phyllotactic transitions. J Theor Biol 139:359-368. https://doi. org/10.1016/S0022-5193(89)80214-0

Meyer HM, Roeder AHK (2014) Stochasticity in plant cellular growth and patterning. Front Plant Sci 5:420. https://doi.org/ 10.3389/fpls.2014.00420

Mirabet V, Besnard F, Vernoux T, Boudaoud A (2012) Noise and robustness in phyllotaxis. PLoS Comput Biol 8:e1002389. https://doi.org/10.1371/journal.pcbi.1002389

Monniaux M, Pieper B, Hay A (2016) Stochastic variation in Cardamine hirsuta petal number. Ann Bot 117:881-887. https:// doi.org/10.1093/aob/mcv131

Monniaux M, Pieper B, McKim SM et al (2018) The role of APETALA1 in petal number robustness. Elife 7:e39399. https:// doi.org/10.7554/eLife.39399

Müller R, Borghi L, Kwiatkowska D et al (2006) Dynamic and compensatory responses of Arabidopsis shoot and floral meristems to CLV3 signaling. Plant Cell 18:1188-1198. https://doi.org/ 10.1105/tpc.105.040444

Nakagawa A, Kitazawa MS, Fujimoto K (2020) A design principle for floral organ number and arrangement in flowers with bilateral symmetry. Development. https://doi.org/10.1242/dev. 182907

Norberg M, Holmlund M, Nilsson O (2005) The BLADE ON PETIOLE genes act redundantly to control the growth and development of lateral organs. Development 132:2203-2213. https://doi.org/10. 1242/dev.01815

Peaucelle A, Couder Y (2016) Fibonacci spirals in a brown alga [Sargassum muticum (Yendo) Fensholt] and in a land plant [Arabidopsis thaliana (L.) Heynh.]: a case of morphogenetic convergence. Acta Soc Bot Pol 85:4. https://doi.org/10.5586/asbp.3526

Peaucelle A, Morin H, Traas J, Laufs P (2007) Plants expressing a miR164-resistant $C U C 2$ gene reveal the importance of post-meristematic maintenance of phyllotaxy in Arabidopsis. Development 134:1045-1050. https://doi.org/10.1242/dev.02774

Peaucelle A, Louvet R, Johansen JN et al (2011) The transcription factor BELLRINGER modulates phyllotaxis by regulating the expression of a pectin methylesterase in Arabidopsis. Development 138:4733-4741. https://doi.org/10.1242/dev.072496

Pelaz S, Ditta GS, Baumann E et al (2000) B and C floral organ identity functions require SEPALLATA MADS-box genes. Nature 405:200-203. https://doi.org/10.1038/35012103

Pieper B, Monniaux M, Hay A (2016) The genetic architecture of petal number in Cardamine hirsuta. New Phytol 209:395-406. https:// doi.org/10.1111/nph.13586 (PMID: 26268614)

Pinon V, Prasad K, Grigg SP et al (2013) Local auxin biosynthesis regulation by PLETHORA transcription factors controls phyllotaxis in Arabidopsis. Proc Natl Acad Sci 110:1107-1112. https:// doi.org/10.1073/pnas.1213497110

Prasad K, Grigg SP, Barkoulas M et al (2011) Arabidopsis PLETHORA transcription factors control phyllotaxis. Curr Biol 21:1123-1128. https://doi.org/10.1016/j.cub.2011.05.009

Raj A, van Oudenaarden A (2008) Nature, nurture, or chance: stochastic gene expression and its consequences. Cell 135:216-226. https://doi.org/10.1016/j.cell.2008.09.050

Raser JM, O'Shea EK (2005) Noise in gene expression: origins, consequences, and control. Science 309:2010-2013. https://doi.org/ 10.1126/science. 1105891

Rebocho AB, Kennaway JR, Bangham JA, Coen E (2017) Formation and shaping of the Antirrhinum flower through modulation of the CUP boundary gene. Curr Biol 27:2610-2622. https://doi.org/ 10.1016/j.cub.2017.07.064

Refahi Y, Brunoud G, Farcot E et al (2016) A stochastic multicellular model identifies biological watermarks from disorders in selforganized patterns of phyllotaxis. Elife 5:e14093. https://doi.org/ 10.7554/eLife. 14093

Reinhardt D, Pesce E-R, Stieger P et al (2003) Regulation of phyllotaxis by polar auxin transport. Nature 426:255-260. https://doi. org/10.1038/nature02081

Ren Y, Chang H-L, Endress PK (2010) Floral development in Anemoneae (Ranunculaceae). Bot J Linn Soc 162:77-100. https://doi. org/10.1111/j.1095-8339.2009.01017.x

Ren Y, Gu T-Q, Chang H-L (2011) Floral development of Dichocarpum, Thalictrum, and Aquilegia (Thalictroideae, Ranunculaceae). Plant Syst Evol 292(3):203-213. https://doi.org/10.1007/ s00606-010-0399-6

Roeder AHK (2018) Use it or average it: stochasticity in plant development. Curr Opin Plant Biol 41:8-15. https://doi.org/10.1016/j. pbi.2017.07.010

Roeder AHK, Chickarmane V, Cunha A et al (2010) Variability in the control of cell division underlies sepal epidermal patterning in Arabidopsis thaliana. PLoS Biol 8:e1000367. https://doi.org/10. 1371/journal.pbio.1000367

Roeder AHK, Cunha A, Ohno CK, Meyerowitz EM (2012) Cell cycle regulates cell type in the Arabidopsis sepal. Development 139:4416-4427. https://doi.org/10.1242/dev.082925

Ronse De Craene LP (2010) Floral diagrams: an aid to understanding flower morphology and evolution. Cambridge University Press, Cambridge

Ronse De Craene L (2018) Understanding the role of floral development in the evolution of angiosperm flowers: clarifications from a historical and physico-dynamic perspective. J Plant Res 131:367-393. https://doi.org/10.1007/s10265-018-1021-1

Rudall PJ (2010) All in a spin: centrifugal organ formation and floral patterning. Curr Opin Plant Biol 13:108-114. https://doi.org/10. 1016/j.pbi.2009.09.019

Rudall PJ, Bateman RM (2003) Evolutionary change in flowers and inflorescences: evidence from naturally occurring terata. Trends Plant Sci 8:76-82. https://doi.org/10.1016/S1360-1385(02) 00026-2

Rümpler F, Theißen G (2019) Reconstructing the ancestral flower of extant angiosperms: the "war of the whorls" is heating up. J Exp Bot 70:2615-2622. https://doi.org/10.1093/jxb/erz106 
Rutishauser R (2016) Acacia (wattle) and Cananga (ylang-ylang): from spiral to whorled and irregular (chaotic) phyllotactic patterns-a pictorial report. Acta Soc Bot Pol 85:3531. https://doi.org/10. 5586/asbp. 3531

Sauquet H, von Balthazar M, Magallón S et al (2017) The ancestral flower of angiosperms and its early diversification. Nat Commun 8:16047. https://doi.org/10.1038/ncomms16047

Sauquet H, von Balthazar M, Doyle JA et al (2018) Challenges and questions in reconstructing the ancestral flower of angiosperms: A reply to Sokoloff et al. Am J Bot 105:127-135. https://doi.org/ 10.1002/ajb2.1023

Schoof H, Lenhard M, Haecker A et al (2000) The stem cell population of Arabidopsis shoot meristems is maintained by a regulatory loop between the CLAVATA and WUSCHEL genes. Cell 100:635-644. https://doi.org/10.1016/S0092-8674(00)80700-X

Smith RS, Guyomarch S, Mandel T et al (2006) A plausible model of phyllotaxis. Proc Natl Acad Sci 103:1301-1306. https://doi.org/ 10.1073/pnas.0510457103

Smyth DR (2018) Evolution and genetic control of the floral ground plan. New Phytol 220:70-86. https://doi.org/10.1111/nph.15282

Sokoloff DD, Remizowa MV, Bateman RM, Rudall PJ (2018) Was the ancestral angiosperm flower whorled throughout? Am J Bot 105:5-15. https://doi.org/10.1002/ajb2.1003

Soltis DE, Chanderbali AS, Kim S et al (2007) The ABC model and its applicability to basal angiosperms. Ann Bot 100:155-163. https://doi.org/10.1093/aob/mcm117

Somssich M, Je BI, Simon R, Jackson D (2016) CLAVATAWUSCHEL signaling in the shoot meristem. Development 143:3238-3248. https://doi.org/10.1242/dev.133645

Stevens PT, Huether CA, Wilson TK (1972) Apical size in the determination of corolla lobe number in Linanthus androsaecus ssp. androsaecus. Am J Bot 59:989-992

Tucker SC (2003) Floral development in legumes. Plant Physiol 131:911-926. https://doi.org/10.1104/pp.102.017459

Uyttewaal M, Burian A, Alim K et al (2012) Mechanical stress acts via katanin to amplify differences in growth rate between adjacent cells in Arabidopsis. Cell 149:439-451. https://doi.org/10.1016/j. cell.2012.02.048

Voelckel C, Borevitz JO, Kramer EM, Hodges SA (2010) Within and between whorls: comparative transcriptional profiling of Aquilegia and Arabidopsis. PLoS ONE 5:e9735. https://doi.org/10. 1371/journal.pone.0009735
Vogt G (2015) Stochastic developmental variation, an epigenetic source of phenotypic diversity with far-reaching biological consequences. J Biosci 40:159-204. https://doi.org/10.1007/ s12038-015-9506-8

Walter A, Schurr U (2005) Dynamics of leaf and root growth: endogenous control versus environmental impact. Ann Bot 95:891-900. https://doi.org/10.1093/aob/mci103

Wang P, Liao H, Zhang W et al (2015) Flexibility in the structure of spiral flowers and its underlying mechanisms. Nat Plants 2:15188. https://doi.org/10.1038/NPLANTS.2015.188

Weinreb C, Rodriguez-Fraticelli A, Camargo FD, Klein AM (2020) Lineage tracing on transcriptional landscapes links state to fate during differentiation. Science. https://doi.org/10.1126/science. aaw3381

Whitehead H (1902) Variation in the moscatel. Biometrika 2:108-113

Wiss D, Zagórska-Marek B (2012) Geometric parameters of the apical meristem and the quality of phyllotactic patterns in Magnolia flowers. Acta Soc Bot Pol 81:203-216. https://doi.org/10.5586/ asbp. 2012.029

Zagórska-Marek B (1987) Phyllotaxis triangular unit; phyllotactic transitions as the consequences of the apical wedge disclinations in a crystal-like pattern of the units. Acta Soc Bot Pol 56:229-255. https://doi.org/10.5586/asbp.1987.024

Zagórska-Marek B (1994) Phyllotaxic diversity in Magnolia flowers. Acta Soc Bot Pol 63:117. https://doi.org/10.5586/asbp.1994.017

Zagórska-Marek B, Szpak M (2008) Virtual phyllotaxis and real plant model cases. Funct Plant Biol 35:1025-1033. https://doi.org/10. 1071/FP08076

Zechner C, Nerli E, Norden C (2020) Stochasticity and determinism in cell fate decisions. Development 147:dev181495. https://doi. org/10.1242/dev.181495

Zhang L, Chen F, Zhang X et al (2020) The water lily genome and the early evolution of flowering plants. Nature 577:79-84. https:// doi.org/10.1038/s41586-019-1852-5

Publisher's Note Springer Nature remains neutral with regard to jurisdictional claims in published maps and institutional affiliations. 